\title{
BMJ Open Predictive utility of the Activity \\ Measure for Post-Acute Care '6-Clicks' short forms on discharge disposition and effect on readmissions: a retrospective observational cohort study
}

\author{
Melissa Harry, ${ }^{1}$ Theo Woehrle, ${ }^{2}$ Colleen Renier, ${ }^{1}$ Margaret Furcht, ${ }^{3}$ \\ Michelle Enockson (D) ${ }^{4}$
}

To cite: Harry M, Woehrle T, Renier C, et al. Predictive utility of the Activity Measure for Post-Acute Care '6-Clicks' short forms on discharge disposition and effect on readmissions: a retrospective observational cohort study. BMJ Open 2021;11:e044278. doi:10.1136/ bmjopen-2020-044278

- Prepublication history and additional material for this paper is available online. To view these files, please visit the journal online (http://dx.doi.org/10. 1136/bmjopen-2020-044278).

Received 28 August 2020 Revised 04 January 2021 Accepted 05 January 2021

\section{A) Check for updates}

C Author(s) (or their employer(s)) 2021. Re-use permitted under CC BY-NC. No commercial re-use. See rights and permissions. Published by BMJ.

${ }^{1}$ Essentia Institute of Rural Health, Essentia Health, Duluth, Minnesota, USA

${ }^{2}$ Telehealth, Essentia Health, Duluth, Minnesota, USA ${ }^{3}$ Hospitalist Services, Essentia Health, Duluth, Minnesota, USA ${ }^{4}$ Rehab Administration, Essentia Health West Region, Fargo, North Dakota, USA

Correspondence to Michelle Enockson; michelle.enockson@ essentiahealth.org

\section{ABSTRACT}

Objectives To evaluate the predictive utility of the Activity Measure for Post-Acute Care '6-Clicks' daily activity and basic mobility functional assessment short forms on inpatient discharge to home compared with skilled nursing facilities, including by diagnostic group (trauma injury, major lower joint replacement/reattachment, spinal fusion excluding cervical), as well as assess the effect of the short forms on 30-day inpatient readmissions.

Design Retrospective, observational cohort study of electronic health record data.

Setting Five hospitals in a multistate, integrated healthcare system serving a large, rural US population. Participants The population-based adult (age $\geq 18$ ) sample of acute care hospitalised patients receiving rehabilitation services included 10316 patients with 12314 hospital admissions from the year prior to 6-Clicks implementation (1 June 2015-31 May 2016) (pre-6-Clicks cohort) and 10931 patients with 13241 admissions from the year after 6-Clicks implementation (1 January 201731 December 2017) (post-6-Clicks cohort). Patients were admitted for major lower joint replacement/reattachment, spinal fusion excluding cervical, trauma injury or another reason.

Intervention 0ccupational and physical therapist use of 6-Clicks daily activity and basic mobility short forms in the post-6-Clicks cohort.

Primary and secondary outcomes Discharge disposition (home, including to assisted living, or skilled nursing facility, including swing beds) and 30-day inpatient readmissions.

Results Areas under the receiver operating characteristic curve were 0.82-0.92 (daily activity) and 0.87-0.94 (basic mobility) for discharge to home or skilled nursing facilities, with trauma and spinal fusion patients having the highest values. Daily activity and basic mobility standardised positive and negative predictive values were highest for the three diagnostic groups compared with the full study sample. Few significant differences in 30-day readmissions were seen between pre- and post-6-Clicks cohorts.

Conclusions 6-Clicks performed well when distinguishing between discharge home or skilled nursing facilities,
Strengths and limitations of this study

- Few other studies have examined the effects of 6-Clicks on discharges to skilled nursing facilities specifically or on 30-day hospital readmissions.

- Our paper appears to be the first to evaluate the effect of 6-Clicks on discharge disposition with trauma patients.

- We also conducted sensitivity analyses comparing different groupings of discharge disposition that are reported in a supplemental file.

- Our study is limited by its retrospective design, inclusion of only electronic health record data, and including only one multistate, integrated healthcare system.

especially by diagnostic group, supporting use by occupational and physical therapists in discharge planning. Future research could assess where additional intervention or training may reduce 30-day readmissions.

\section{INTRODUCTION}

Correctly identifying discharge destination for acute care patients receiving in hospital rehabilitation has both financial and morbidity implications. In 2018, nursing facility expenditures exceeded US $\$ 123.7$ billion in the USA. ${ }^{1}$ In that same year, US Medicare beneficiaries had approximately 2.2 million skilled nursing facility (SNF) admissions, resulting in US $\$ 28.5$ billion in Medicare costs. $^{2}$ Skilled nursing care after an acute hospitalisation can improve functional status and reduce long-term admissions to nursing homes and mortality, particularly for older adults. ${ }^{34}$ However, based on Medicare claims, the 30 -day readmission rate is $28.6 \%$ from SNFs, suggesting there are opportunities for improved care and cost savings. ${ }^{4}$ Not all patients require an inpatient skilled nursing 
level of care for continuing rehabilitation following an acute hospitalisation. Nevertheless, accurately predicting the most appropriate discharge disposition remains a challenge.

Functional status plays an important role in determining the necessary level of care following an acute hospitalisation. Recent evidence supports the use of the Activity Measure for Post-Acute Care (AM-PAC) ${ }^{5} 6$ '6-Clicks' inpatient daily activity and basic mobility short forms, which are standardised functional assessment tools for predicting the discharge disposition of medical and surgical hospital inpatients. ${ }^{7-15}$ 6-Clicks assesses two functional domains: daily activities (eg, self-care, such as dressing and toileting) and basic mobility (eg, movement, such as walking and moving from one position to another)..$^{912}$ Items represent types of activities occupational and physical therapists routinely assess in acute care settings and those important when evaluating patients for postacute care. ${ }^{10}$ The 6-Clicks tools are brief, easy to use and provide transparent measures of patients' functional status, allowing occupational and physical therapists to identify therapy needs and assist in patientcentred discharge planning. ${ }^{9} 10$ The tools have also been successfully translated into other languages. ${ }^{16-18}$

\section{OBJECTIVE AND AIMS}

As part of a quality improvement initiative to standardise functional assessment and enhance discharge planning, an integrated healthcare system implemented the AM-PAC 6-Clicks daily activity and basic mobility (V.2) short forms for hospitalised patients receiving inpatient rehabilitation within five healthcare system hospitals. Occupational therapists collect daily activity data, while physical therapists collect basic mobility data to inform discharge planning and discharge recommendations.

The objective of this study was to add to the evidence base by evaluating the utility of 6-Clicks with adult patients who received rehabilitation services during their acute care hospitalisation in one of five healthcare system hospitals. The primary aims were twofold: (1) determine the accuracy of 6-Clicks scale scores in predicting overall discharge disposition between home and SNFs, as little research has focused on discharge to SNFs specifically; ${ }^{8}$ and (2) examine accuracy for three diagnostic groups: patients that were admitted for a trauma-related event, patients admitted after major lower joint replacements or reattachments, and patients admitted after spinal fusions (except cervical). A secondary aim of this study was to assess the impact of 6-Clicks on 30-day postdischarge hospital readmissions within the healthcare system for these patients.

\section{METHODS}

\section{Study population}

The location of this study was Essentia Health, an upper Midwestern integrated healthcare system serving patients

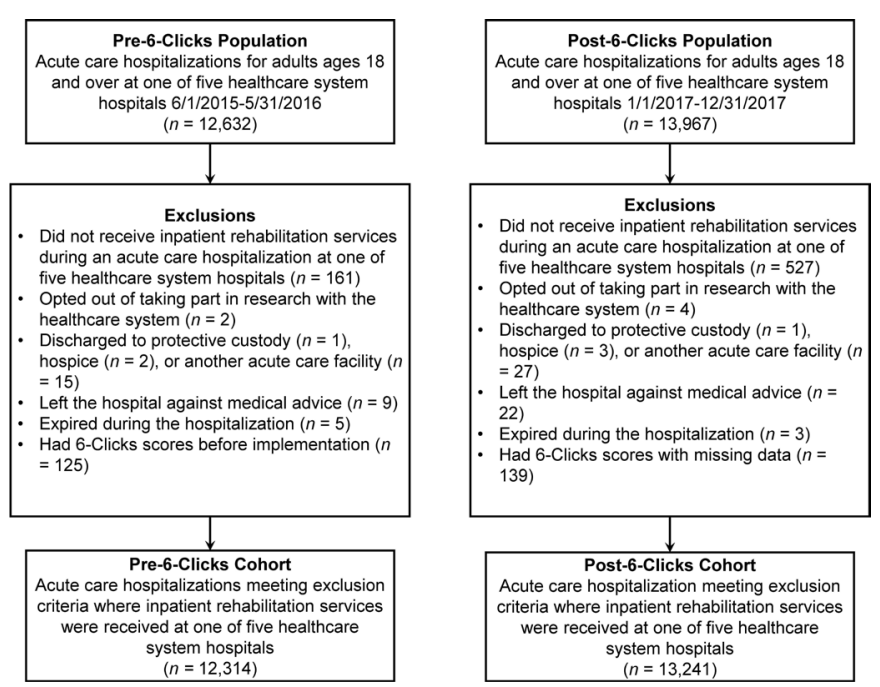

Figure 1 Study inclusion and exclusion criteria flow chart.

throughout rural areas of northern Minnesota, northwestern Wisconsin and eastern North Dakota. The study population included hospitalisations for adult (age $\geq 18$ ) patients who received inpatient rehabilitation services during an acute hospitalisation at one of five healthcare system hospitals from 1 June 2015-31 May 2016, before the hospitals implemented 6-Clicks (pre-6-clicks Cohort, $\mathrm{n}=10316$, with 12314 hospitalisations), and 1 January 2017-31 December 2017, the year following 6-Clicks implementation (post-6-Clicks cohort, $\mathrm{n}=10931$, with 13241 hospitalisations). 6-Clicks was implemented in all five hospitals on 1 January 2017.

Figure 1 presents a flow chart of study inclusion and exclusion criteria by acute care hospitalisation. Of note, we excluded 125 patients in the pre-6-Clicks cohort with 6-Clicks scores (10 had only daily activity, 24 had only basic mobility and 91 both daily activity and basic mobility scores). This was due to some occupational and physical therapists in two hospitals implementing 6-Clicks into practice before all other healthcare system hospitals. Furthermore, we excluded 139 patients where items composing 6-Clicks scores included missing data (daily activity, $n=44$; basic mobility, $n=97$; with two cases missing both scores). These total scores were either miscalculated or calculated correctly by excluding the stairs-related basic mobility question, which can be excluded for some patients. ${ }^{19}$

\section{Patient and public involvement statement}

Due to the retrospective nature of this study, there was no patient or public involvement.

\section{Study design}

We conducted a retrospective observational cohort study from existing electronic health record (EHR) data. We received a waiver of Health Insurance Portability and Accountability Act authorisation and a complete waiver of patient informed consent from the Essentia Health Institutional Review Board. However, patients who had opted 
out of research at Essentia Health were excluded from the study sample (see figure 1).

\section{Instrument}

Both 6-Clicks daily activity and basic mobility response options reflect level of assistance needed to perform each element: total $=1$, a lot $=2$, a little $=3$ and none $=4{ }^{19}$ Total scores range from 6 to 24, with scaled scores used in calculating percent impairment. ${ }^{11}$ In clinical practice at Essentia Health, total scores and percent impairment are calculated in the EHR based on item scores entered into an electronic flowchart by occupational and physical therapists. In this study, scale scores were calculated from total scores to allow for comparison with research by Jette $e t a l^{9}$

Previous research on the reliability of 6-Clicks showed high overall intraclass correlation coefficients in the general acute care patient population (daily activity $=0.783$, basic mobility $=0.849),{ }^{11}$ within an intensive care unit (ICU) patient population (basic mobility $=0.957),{ }^{20}$ and within a neuroscience inpatient department (basic mobility: physical therapist pairs $=0.91$, nurse pairs $=0.97) .{ }^{21}$ However, intraclass correlation coefficient ranges between rater pairs were more variable in the general acute care patient population, as were weighted kappa scores, with more variability seen between occupational therapists than physical therapists. ${ }^{11}$ Both scales have shown good levels of internal consistency reliability (daily activity $=0.91$, basic mobility=0.96) ${ }^{10}$ Regarding predicting discharge disposition comparing home to another institution, Jette et al reported areas under the receiver operating characteristic curve of 0.846 and 0.845 for daily activity scale scores and 0.857 and 0.855 for basic mobility scores in randomly selected development and validation samples. ${ }^{9}$ The authors also reported that scale score cut points of 39.4 (daily activity) and 42.9 (basic mobility) 'provided the highest sensitivity, specificity and positive and negative predictive values (PPV/NPV)' (Jette et al, p. 1258). ${ }^{9}$

\section{Data collection}

We collected standardised EHR data for each eligible patient. Patient outcome data included discharge disposition and 30-day readmissions. We grouped discharge disposition into discharge to home (including with home healthcare services or to assisted living) compared with those discharged to a SNF (including swing beds). In sensitivity analyses (see the online supplemental appendix), we followed Jette et al by grouping discharge disposition into those who were discharged home (including with home healthcare services) compared with those discharged to an institution (including assisted living). ${ }^{9}$ Furthermore, in our sensitivity analyses, we also grouped discharge disposition into those who were discharged home (including with home healthcare services or to assisted living) compared with those discharged to an institution. We grouped assisted living with home in our primary aims and some sensitivity analyses due to the common practice of occupational and physical therapists at the healthcare system recording discharge to assisted living as discharge to home.

Independent variables in multivariate models included: pre- or post-6-Clicks cohort (for cohort comparisons only); patient demographics (age, sex, race, Hispanic ethnicity); primary diagnostic group (elective lower joint replacement or reattachment; spinal fusion not including cervical; other medical diagnosis); trauma-related hospitalisation; admission hospital; number of prior admissions during the study period; number of prior admissions within the past 30 days; length of hospitalisation; Charlson Comorbidity Index score; and 6-Clicks daily activity and basic mobility scale scores (post-6-Clicks cohort only). Unlike Jette $e t$ $a l,{ }^{9}$ who employed initial 6-Clicks scores in analyses, we used final (some of which were initial) 6-Clicks scores as these scores were used in determining discharge recommendations. While Jette et al recommend initial 6-Clicks scores be used in discharge planning, ${ }^{9}$ a patient's condition can change during the course of hospitalisation, requiring reassessment.

\section{Data analysis}

Analyses were conducted in SPSS V.23.0. ${ }^{22}$ For our first primary aim, we assessed the predictive ability of 6-Clicks scale scores by following Jette et al and randomly splitting the post-6-Clicks study cohort in two, calculating area under the receiver operating characteristic curve (AUC) in one half and repeating the analyses in the other for both daily activity and basic mobility scale scores. ${ }^{9}$ In our second primary aim, we split the post-6-Clicks cohort up into three diagnostic groups (trauma-related hospitalisation; elective lower joint replacement or reattachment; spinal fusion not including cervical) and again assessed AUC for both 6-Clicks scale scores. Also like Jette $e t a l$, we assessed model calibration using the Hosmer-Lemeshow goodness of fit test, as well as used multivariate logistic regression to compare and plot predicted to observed discharges for patients' first hospitalisation in the post6-Clicks cohort in our two primary aims and in reporting positive and negative predicted values with a standardised $50 \%$ prevalence for discharge to home. ${ }^{9}$ We also report effect sizes in the form of ORs.

For our secondary aim, the unit of analysis was acute hospitalisation with receipt of inpatient rehabilitation services, as patients could have more than one hospitalisation and be included in both study cohorts. Because there were multiple patients within each hospital, a varying number of hospitalisations per patient, and patients with hospitalisations in both cohorts, these events would be expected to be intercorrelated. As such, in our analyses comparing pre- and post-6-Clicks 30-day readmissions, we employed bivariate and multivariate generalised estimating equations with unstructured correlation matrices that included events nested within patients nested within hospitals. 
Table 1 Sample descriptive statistics by study cohort

\begin{tabular}{|c|c|c|}
\hline & Pre-6-Clicks & Post-6-Clicks \\
\hline Unique patients & 10316 & 10931 \\
\hline Female & $5436(52.7 \%)$ & $5662(51.8 \%)$ \\
\hline \multicolumn{3}{|l|}{ Race } \\
\hline White & $9779(94.8 \%)$ & $10273(94.0 \%)$ \\
\hline American Indian & $377(3.7 \%)$ & $446(4.1 \%)$ \\
\hline Black & $73(0.7 \%)$ & $105(1.0 \%)$ \\
\hline Asian & $18(0.2 \%)$ & $22(0.2 \%)$ \\
\hline Native Hawaiian/Pacific Islander & $14(0.1 \%)$ & $15(0.1 \%)$ \\
\hline Unknown & $55(0.5 \%)$ & $70(0.6 \%)$ \\
\hline Hispanic ethnicity & $34(0.3 \%)$ & $50(0.5 \%)$ \\
\hline Unknown & $31(0.3 \%)$ & $52(0.5 \%)$ \\
\hline Total hospitalisations per patient, n, Mdn, IQR, range & $12314,1,1-1,1-8$ & $13241,1,1-1,1-8$ \\
\hline \multicolumn{3}{|l|}{ Hospitalisations per healthcare system hospital } \\
\hline Hospital 1 & $6210(50.4 \%)$ & $6868(51.9 \%)$ \\
\hline Hospital 2 & $2897(23.5 \%)$ & $3029(22.9 \%)$ \\
\hline Hospital 3 & $1854(15.1 \%)$ & $2032(15.3 \%)$ \\
\hline Hospital 4 & $804(6.5 \%)$ & $797(6.0 \%)$ \\
\hline Hospital 5 & 549 (4.5\%) & $515(3.9 \%)$ \\
\hline Patient age at unique hospitalisation: Mdn, IQR, range & $72,61-81,18-106$ & $71,61-81,18-104$ \\
\hline Length of stay in days: Mdn, IQR, range & $4,2-6,0-102$ & $4,2-6,0-128$ \\
\hline Charlson Comorbidity Index score: Mdn, IQR, range & $0,0-1,0-12$ & $0,0-1,0-14$ \\
\hline Prior admissions in study: Mdn, IQR, range & $0,0-0,0-7$ & $0,0-1,0-10$ \\
\hline Prior admissions in past 30 days: Mdn, IQR, range & $0,0-0,0-3$ & $0,0-0,0-3$ \\
\hline 30-day inpatient readmissions: Mdn, IQR, range & $0,0-0,0-5$ & $0,0-0,0-4$ \\
\hline \multicolumn{3}{|l|}{ Hospitalisation cause } \\
\hline Traumatic injury* & $818(6.6 \%)$ & $811(6.1 \%)$ \\
\hline Specific procedure & $2374(19.3 \%)$ & $2446(18.5 \%)$ \\
\hline Spinal fusion except cervical & $215(9.1 \%)$ & $267(10.9 \%)$ \\
\hline Major lower extremity joint replacement or reattachment & $2159(90.9 \%)$ & $2179(89.1 \%)$ \\
\hline Other cause & $9190(74.6 \%)$ & $10072(76.1 \%)$ \\
\hline \multicolumn{3}{|l|}{ Discharge disposition } \\
\hline Home with or without services & $8366(67.9 \%)$ & $9073(68.5 \%)$ \\
\hline Intermediate care facility/assisted living & $356(2.9 \%)$ & $376(2.8 \%)$ \\
\hline Skilled nursing facilities/swing bed & $3544(28.8 \%)$ & $3761(28.4 \%)$ \\
\hline Another healthcare institution & $48(0.4 \%)$ & $31(0.2 \%)$ \\
\hline Daily activity 6-Clicks scale score: M (SD) & $\mathrm{N} / \mathrm{A}$ & $42.56(7.57)$ \\
\hline Percent impairment: M (SD) & N/A & $37.02 \%(18.71)$ \\
\hline Basic mobility 6-Clicks scale score: M (SD) & $\mathrm{N} / \mathrm{A}$ & $48.34(8.79)$ \\
\hline Percent impairment: M (SD) & N/A & $32.66 \%(20.27)$ \\
\hline
\end{tabular}

*Patients could have a traumatic hospitalisation associated with either a spinal fusion except cervical or a major lower extremity joint replacement or reattachment.

M, mean; Mdn, median; N/A, not applicable.

\section{RESULTS}

A total of 25555 hospitalisations for 21247 adult (18 and over) patients met inclusion criteria for this study. Table 1 presents descriptive statistics for the pre- and post-6-Clicks cohorts. The majority of patients were white, female and discharged to home with or without home healthcare, 
followed by discharges to SNFs. Median length of stay was 4 days in both cohorts. Median patient age was 72 in the pre-6-Clicks cohort and 71 in the post-6-Clicks cohort. Patients were admitted for major lower joint replacement or reattachment, spinal fusion excluding cervical, trauma injury or another reason and received rehabilitation services during their acute care hospitalisation.

\section{Predictive utility of 6-Clicks scores in determining patient discharge disposition \\ Full sample}

For discharge to home compared with SNF, daily activity AUC ranged from 0.84 to 0.85 , while basic mobility AUC was slightly higher at 0.87 (95\% CI 0.86 to 0.88 ) in development and validation samples (table 2). Standardised PPVs for home discharge were slightly higher than NPVs. Each one-point increase in either score significantly increased the odds of discharge to home for the full sample patients. The Hosmer-Lemeshow tests were significant $(p<0.001)$ for each sample and scale score, suggesting lack of model fit. However, bivariate models and sample size may have affected significance levels. Sensitivity analyses presented in online supplemental table S1 illustrate highly similar or the same results as in the main analyses.

Online supplemental figure 1 presents stacked scatter plots for discharge to home versus SNFs. Jette $e t$ als cut points of 39.4 and 42.9 for daily activity and basic mobility scale scores had lower specificity than sensitivity in both development (daily activity sensitivity 0.834 and specificity 0.701 ; basic mobility sensitivity 0.889 and specificity 0.652 ) and validation (daily activity sensitivity 0.824 and specificity 0.723 ; basic mobility sensitively 0.882 and specificity 0.653 ) (not shown in table 2) samples. ${ }^{9}$ A slightly higher basic mobility cut point of 44.5 presented higher specificity at the expense of slightly slower sensitivity (development sample: sensitivity 0.803 and specificity 0.754 . Validation sample: sensitivity 0.797 and specificity 0.759) (not shown in table 2).

We next assessed the predictive utility of 6-Clicks scores in multivariate logistic regression models including only the first hospitalisation event for the post-6-Clicks cohort for the full sample (table 3). Of note, a one-point increase in daily activity and basic mobility scale scores was significantly associated with a higher likelihood of patients being discharged home regardless of model. Similar results were found in sensitivity analyses (online supplemental tables S2 and S3). Hosmer-Lemeshow tests were all significant, suggesting lack of model fit.

\section{Diagnostic groups}

As shown in table 4, daily activity AUC were 0.90 or greater for trauma $(0.90,95 \%$ CI 0.88 to 0.93$)$ and spinal fusion patients $(0.92,95 \%$ CI 0.88 to 0.97$)$, although AUC was 0.82 (95\% CI 0.79 to 0.85 ) for major lower joint replacements or reattachments. Basic mobility AUC was 0.90 or greater for each of the three diagnostic groups, where it was greatest for spinal fusions $(0.94,95 \%$ CI 0.90 to 0.97$)$, followed by trauma $(0.92$, $95 \%$ CI 0.90 to 0.94 ), then major lower joint replacements or reattachments $(0.91,95 \%$ CI 0.89 to 0.92$)$. Specificity 
Table 3 Multivariate logistic regression models assessing predictive utility of 6-Clicks daily activity and basic mobility scale scores for discharge home with or without services (including assisted living) versus skilled nursing facilities*

\begin{tabular}{|c|c|c|c|c|c|c|c|c|c|c|c|}
\hline \multirow{2}{*}{$\begin{array}{l}\text { Models and } \\
\text { groups }\end{array}$} & \multirow[b]{2}{*}{$\mathbf{n}$} & \multirow[b]{2}{*}{$\mathbf{R}^{2} \dagger$} & \multirow[b]{2}{*}{$\mathbf{R}^{2} \ddagger$} & \multirow[b]{2}{*}{$\chi^{2}(\mathrm{df}) \S$} & \multirow[b]{2}{*}{ P Value§ } & \multicolumn{3}{|c|}{ Daily activity } & \multicolumn{3}{|c|}{ Basic mobility } \\
\hline & & & & & & OR & $95 \% \mathrm{Cl}$ & $P$ value & OR & $95 \% \mathrm{Cl}$ & $P$ value \\
\hline \multicolumn{12}{|l|}{ Full sampleף } \\
\hline Basic mobility & 8734 & 0.35 & 0.52 & $29.40(8)$ & $<0.001$ & N/A & N/A & N/A & 1.22 & 1.21 to 1.24 & $<0.001$ \\
\hline $\begin{array}{l}\text { Daily activity and } \\
\text { basic mobility }\end{array}$ & 7321 & 0.39 & 0.57 & $54.57(8)$ & $<0.001$ & 1.14 & 1.12 to 1.16 & $<0.001$ & 1.14 & 1.13 to 1.16 & $<0.001$ \\
\hline \multicolumn{12}{|l|}{ Trauma** } \\
\hline Daily activity & 560 & 0.49 & 0.67 & $16.56(8)$ & 0.035 & 1.33 & 1.25 to 1.41 & $<0.001$ & $\mathrm{~N} / \mathrm{A}$ & N/A & N/A \\
\hline Basic mobility & 583 & 0.52 & 0.71 & $14.01(8)$ & 0.082 & N/A & N/A & N/A & 1.30 & 1.23 to 1.37 & $<0.001$ \\
\hline $\begin{array}{l}\text { Daily activity and } \\
\text { basic mobility }\end{array}$ & 535 & 0.55 & 0.76 & $7.68(8)$ & 0.465 & 1.22 & 1.12 to 1.32 & $<0.001$ & 1.22 & 1.14 to 1.31 & $<0.001$ \\
\hline $\begin{array}{l}\text { Daily activity and } \\
\text { basic mobility }\end{array}$ & 1731 & 0.37 & 0.68 & $2.90(8)$ & 0.941 & 1.29 & 1.20 to 1.38 & $<0.001$ & 1.27 & 1.22 to 1.32 & $<0.001$ \\
\hline \multicolumn{12}{|c|}{ Spinal fusion excluding cervical ${ }^{\star \star}$} \\
\hline Daily activity & 238 & 0.43 & 0.70 & $2.83(8)$ & 0.945 & 1.92 & 1.55 to 2.39 & $<0.001$ & $\mathrm{~N} / \mathrm{A}$ & N/A & N/A \\
\hline Basic mobility & 228 & 0.45 & 0.72 & $46.48(8)$ & $<0.001$ & N/A & N/A & N/A & 1.71 & 1.38 to 2.12 & $<0.001$ \\
\hline $\begin{array}{l}\text { Daily activity and } \\
\text { basic mobility }\end{array}$ & 226 & 0.50 & 0.80 & $45.53(8)$ & $<0.001$ & 1.59 & 1.24 to 2.03 & $<0.001$ & 1.47 & 1.20 to 1.80 & $<0.001$ \\
\hline
\end{tabular}

${ }^{*}$ Events include first events only.

†Cox and Snell $R^{2}$.

$\ddagger$ Nagelkerke $R^{2}$.

§Hosmer-Lemeshow test.

ๆControlling for age, gender, race, Hispanic ethnicity, hospital, Charlson Comorbidity Index score, number of previous admissions in the study period (including in the pre-6-Clicks cohort), number of previous admissions in the last 30 days (including in the pre-6-Clicks cohort), primary diagnosis type (major lower extremity replacement or reattachment, spinal fusion except cervical, or other), and whether or not the hospitalisation was due to trauma.

${ }^{*}$ Controlling for age, gender, race, Hispanic ethnicity, hospital, Charlson Comorbidity Index score, number of previous admissions in the study period (including in the pre-6-Clicks cohort). Number of previous admissions in the last 30 days was excluded due to zero values in the diagnostic group analyses.

NA, not applicable.

exceeded sensitivity in all models. Standardised PPVs were also higher than standardised NPVs for each group, but the highest values were seen for lower major joint replacementor reattachment patients, followed by spinal fusions excluding cervical, then patients with trauma. Sensitivity analyses in online supplemental tables S4 and S5 present similar results.

We next evaluated the predictive utility of 6-Clicks scale scores by diagnostic groups (table 3), showing that like in the full model, a one-point increase in either daily activity, basic mobility, or both scores together significantly increased the odds of patients being discharged home for all diagnostic groups and models. Most models, but not all, showed improved fits compared with the full sample and had nonsignificant Hosmer-Lemeshow tests. Similar results were found in sensitivity analyses (online supplemental tables S2 and S3).

\section{6-Clicks effect on 30-day postdischarge inpatient readmissions}

When assessing the impact of 6-Clicks on 30-day postdischarge inpatient readmissions by individual hospital and all hospitals together, only a few statistically significant differences were noted between pre- and post-6-Clicks cohorts (table 5). While not significant in the bivariate models for hospital 1, being in the post-6-Clicks cohort was significantly associated with an incident rate ratio $0.90(95 \%$ CI 0.81 to $0.99, \mathrm{p}=0.030$ ) times that of the pre-6-Clicks cohort in the log count of 30-day inpatient readmissions. For hospital 4 in both bivariate and multivariate models, being in the post-6Clicks cohort was significantly associated with incident rate ratios 1.47 (95\% CI 1.06 to $2.03, \mathrm{p}=0.021)$ and 1.37 (95\% CI 1.00 to $1.88, \mathrm{p}=0.048$ ) times the pre-6-Clicks cohort. No significant differences in readmissions were seen for all hospitals together. Hospital discharges to home versus SNFs did differ significantly in each study cohort (online supplemental table S6), with few significant differences seen within hospitals preand post-6-Clicks (online supplemental table S7). 


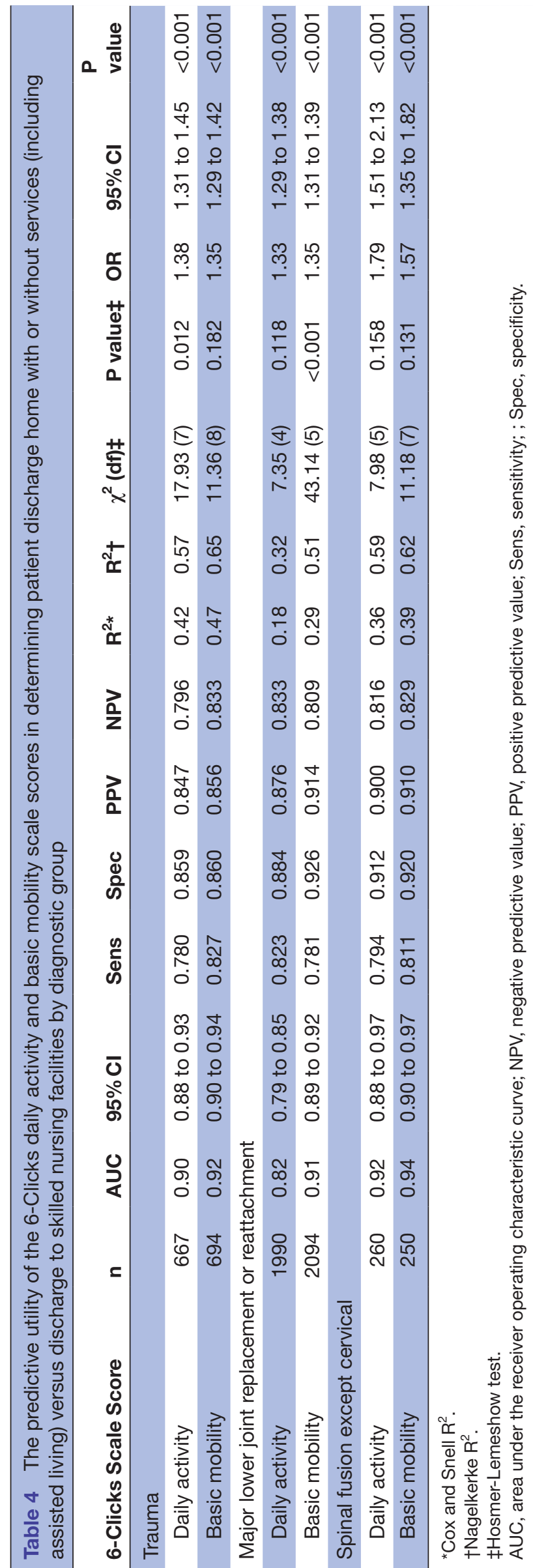

\section{DISCUSSION}

In this study, we evaluated the AM-PAC '6-Clicks' inpatient daily activity and basic mobility short forms ${ }^{5-11}$ with acute care hospitalised patients receiving rehabilitation services in one of five hospitals in the Essentia Health integrated healthcare system. Our findings showed that daily activity and basic mobility AUC ranges for discharge to home (including with home healthcare services and to assisted living) or SNFs (including swing beds) for the full sample were similar to some previous research, ${ }^{913}$ but higher than both the full sample and other research when focusing on diagnostic groups (trauma, major lower joint replacement or reattachment, spinal fusion excluding cervical) ${ }^{14}$ However, using Jette $e t a l$ s cut points of 39.4 and 42.9 for daily activity and basic mobility scale scores provided different levels of sensitivity and specificity when considering discharge to home versus SNFs, or other institutions in our sensitivity analyses, than previously reported. ${ }^{9}$ We also found lower sensitivity, higher specificity, and somewhat varied PPV and NPV for 6-Clicks scores than Jette $e t a l^{9}$ with a slightly higher cut point score of 44.5 for basic mobility showing increased sensitivity and slightly lowered specificity. Finally, few significant impacts were seen on 30-day readmissions between pre- and post-6-Clicks cohorts. Hospitals did differ generally in discharge dispositions, with few differences seen within hospitals between pre- and post-6-Clicks cohorts. Previous research has found that level of hospital rurality may affect receipt of postacute care. ${ }^{23}$ With the exception of strokes, patients in rural hospitals have been shown to have significantly lower postacute care receipt of skilled nursing and home healthcare compared with patients discharged from urban hospitals. ${ }^{23}$

Our findings were unique in that while we included the comparison of home versus institution as reported by Jette $e t$ $a l$ in our sensitivity analyses, ${ }^{9}$ we also assessed two additional combinations of discharge to home or another institution or to SNFs, finding little differences between these combinations. This result is important to note in practical terms, as discharge disposition may be unclear in the medical record. For example, an individual living in assisted living may be coded as discharged to home rather than to an institution. Furthermore, our study followed Jette $e t$ al and included all acute care hospitalisations, ${ }^{9}$ whether hip, knee, or spinal surgeries, other causes, and/or trauma-related injuries, factors which we controlled for in multivariate models. Other recent research has also focused on specific diagnostic, unit or procedure groups, ${ }^{78121415}$ which we followed by focusing on diagnostic groups in our analyses, specifically trauma events, lower major joint replacements or reattachments and spinal fusions excluding cervical.

For our full sample, we found higher AUC for 6-Clicks scores related to discharge disposition to SNFs or to institutions generally than a number of recent studies. This includes findings from a study with adults age 55 and over with cardiovascular disease, ${ }^{7}$ as well as research with general acute care patients receiving rehabilitation ${ }^{13}{ }^{15}$ and patients in ICUs. ${ }^{14}$ Our results also showed higher AUC for our diagnostic groups of patients admitted for trauma, spinal fusions excluding cervical or major lower joint replacements or 
Table 5 Bivariate and multivariate generalised estimating equations: hospital 30-day postdischarge inpatient readmissions (yes/no) and counts by hospital and overall

\begin{tabular}{|c|c|c|c|c|}
\hline \multirow[b]{2}{*}{ Models } & \multicolumn{2}{|c|}{ 30-day hospitalisation yes/no* } & \multicolumn{2}{|c|}{ 30-day inpatient readmission counts } \\
\hline & OR $(95 \% \mathrm{Cl})$ & $P$ value & IRR (95\% CI) & $P$ value \\
\hline \multicolumn{5}{|l|}{ Hospital $1 \ddagger$} \\
\hline Bivariate§ & 0.97 (0.87 to 1.08$)$ & 0.561 & 0.94 (0.84 to 1.04$)$ & 0.233 \\
\hline Multivariate & $0.92(0.84 \text { to } 1.01)^{\star \star}$ & 0.065 & $0.90(0.81 \text { to } 0.99)^{\star *}$ & 0.030 \\
\hline \multicolumn{5}{|l|}{ Hospital 2} \\
\hline Bivariate§ & $1.11(0.95$ to 1.30$)$ & 0.201 & 1.12 (0.95 to 1.31$)$ & 0.168 \\
\hline Multivariate & $1.02(0.88$ to 1.20$) \dagger \dagger$ & 0.769 & 1.03 (0.89 to 1.19$) \dagger \dagger$ & 0.727 \\
\hline \multicolumn{5}{|l|}{ Hospital 3} \\
\hline Bivariate§ & 1.05 (0.83 to 1.32$)$ & 0.704 & 1.01 (0.80 to 1.28$)$ & 0.916 \\
\hline Multivariate & $1.01(0.82$ to 1.25$)$ & 0.704 & 0.98 (0.81 to 1.18$)$ & 0.814 \\
\hline \multicolumn{5}{|l|}{ Hospital 4} \\
\hline Bivariate§ & 1.37 (0.99 to 1.91$)$ & 0.060 & 1.47 (1.06 to 2.03$)$ & 0.021 \\
\hline Multivariate & $1.28(0.93 \text { to } 1.77)^{\star \star}$ & 0.129 & $1.37(1.00 \text { to } 1.88)^{\star \star}$ & 0.048 \\
\hline \multicolumn{5}{|l|}{ Hospital 5} \\
\hline Bivariate§ & 0.99 (0.68 to 1.45$)$ & 0.976 & 0.98 (0.69 to 1.39$)$ & 0.920 \\
\hline Multivariate & $0.87(0.60 \text { to } 1.25)^{\star *}$ & 0.448 & $0.88(0.63 \text { to } 1.23)^{\star *}$ & 0.460 \\
\hline \multicolumn{5}{|l|}{ All Hospitalsł‡ } \\
\hline Bivariate§ & 1.03 (0.95 to 1.12$)$ & 0.444 & 1.01 (0.94 to 1.10$)$ & 0.743 \\
\hline Multivariateף & 0.96 (0.89 to 1.04$) \neq \ddagger$ & 0.324 & 0.96 (0.89 to 1.03$) \neq \ddagger$ & 0.220 \\
\hline
\end{tabular}

*Negative binomial. All models had lower Quasi-likelihood under Independence Model Criterion (QIC) and Corrected Quasi-likelihood under Independence Model Criterion (QICC) than Poisson generalised estimating equations (not shown).

†Binary logit link.

$\ddagger$ The indicator variable for trauma patients was only included in multivariate models including Hospital 1.

§Compared to the pre-6-Clicks cohort.

ПCompared to the pre-6-Clicks cohort. Multivariate models also controlled for gender, race, Hispanic ethnicity, age, hospital, Charlson Comorbidity Index score, whether the admission was related to a trauma event, diagnosis type (major lower joint replacement or reattachment, spinal fusion excluding cervical, other), prior admissions in the study, and prior admissions in the last 30 days.

**Hispanic ethnicity and race removed from the model due singular Hessian matrices resulting in uncertainty of valid model fit or unexpected estimation error.

††Race removed from the model due to singular Hessian matrix or unexpected estimation error.

¥¥Hispanic ethnicity was removed from the model due to lack of convergence, singular Hessian matrix or unexpected estimation error. IRR, incidence rate ratio.

reattachments than either our full sample or other research for both discharge to SNFs, as well as to other institutions. $^{7913-15}$

Future research could confirm our findings with other populations. Research could also assess whether additional training or EHR improvements may optimise 6-Clicks use in discharge planning and reducing 30-day readmissions. Lastly, research could further assess therapists' experiences with the adoption and use of 6-Clicks. ${ }^{24} \mathrm{~A}$ previous qualitative study on the perceptions of occupational and physical therapists about 6-Clicks did show hesitancy to fully embrace these tools in practice for a variety of reasons. ${ }^{24}$ Therapists also reported that while they documented 6-Clicks for patients as a mandatory part of clinical care, they did not actually use 6-Clicks scores. ${ }^{24}$ Research has shown high levels of interrater reliability, ${ }^{11}{ }^{20}$ as well as illustrating areas where rater agreement was low, ${ }^{11}$ lending some credence to therapists' distrust in the scoring system. ${ }^{24}$

\section{Limitations}

Our study was limited in that it only included one healthcare system and involved retrospective review of data collected for clinical care. We were also unable to capture 30 -day postdischarge admissions outside the healthcare system. In addition, some occupational and physical therapists in two healthcare system hospitals began using the tools prior to implementation. These early adopters had more experience with the tools, although all occupational and physical therapists received the same system-wide training. Moreover, there was a $2.4 \%$ error rate in discharge disposition in the EHR. When assessing a random sample of $1 \%(\mathrm{n}=250)$ of all hospitalisations in the study, we initially identified a $7.2 \%(n=18)$ error rate. Eleven errors were patients recorded as discharged to home or home with healthcare services when discharge was the opposite. One error was when a patient recorded as discharged home was discharged to assisted living, the patient's residence prior to hospitalisation. We addressed these errors by combining 
discharges home, home with healthcare services and assisted living in our primary analyses. Six errors were patients documented as discharged home when discharge was actually to SNFs $(n=5)$ or inpatient rehabilitation $(n=1)$, leaving an unaddressed 2.4\% EHR discharge disposition error rate.

Finally, we were unable to control for how occupational and physical therapists used the 6-Clicks short forms in discharge planning in our models. Future research could assess this area.

\section{CONCLUSIONS}

AM-PAC '6-Clicks' daily activity and basic mobility short forms ${ }^{5-11}$ performed best with patient groups (trauma, major lower joint replacements or reattachments and spinal fusions excluding cervical) when determining discharge to home or SNF in this study. Our findings support the use of 6-Clicks in occupational and physical therapists' discharge planning, particularly for patients being discharged to home. Thirty-day hospital readmissions showed little significant change between pre- and post-6-Clicks cohorts, suggesting areas for targeted improvement within the healthcare system. More research is needed to determine if our findings, including regarding 30-day hospital readmissions and diagnostic groups, are generalisable outside of the healthcare system.

Acknowledgements The authors thank Austin Land, Essentia Institute of Rural Health Research Informatics Analyst II, for assistance with data collection.

Contributors $\mathrm{MH}$ drafted the manuscript, developed the data analysis plan, and analysed the data. TW contributed to the literature review and managed the project. $\mathrm{CR}$ developed the data analysis plan. MF helped develop the study protocol. ME led the study, development of the study protocol, and guided the study aims. All authors contributed to the manuscript and approved of the final version.

Funding The authors have not declared a specific grant for this research from any funding agency in the public, commercial or not-for-profit sectors.

Competing interests None declared.

Patient consent for publication Not required.

Ethics approval This study was reviewed, approved and monitored by the Essentia Health Institutional Review Board (approval number EH18545).

Provenance and peer review Not commissioned; externally peer reviewed.

Data availability statement Deidentified data are available from the corresponding author on reasonable request.

Supplemental material This content has been supplied by the author(s). It has not been vetted by BMJ Publishing Group Limited (BMJ) and may not have been peer-reviewed. Any opinions or recommendations discussed are solely those of the author(s) and are not endorsed by BMJ. BMJ disclaims all liability and responsibility arising from any reliance placed on the content. Where the content includes any translated material, BMJ does not warrant the accuracy and reliability of the translations (including but not limited to local regulations, clinical guidelines, terminology, drug names and drug dosages), and is not responsible for any error and/or omissions arising from translation and adaptation or otherwise.

Open access This is an open access article distributed in accordance with the Creative Commons Attribution Non Commercial (CC BY-NC 4.0) license, which permits others to distribute, remix, adapt, build upon this work non-commercially, and license their derivative works on different terms, provided the original work is properly cited, appropriate credit is given, any changes made indicated, and the use is non-commercial. See: http://creativecommons.org/licenses/by-nc/4.0/.

ORCID iD

Michelle Enockson http://orcid.org/0000-0002-1069-1066
REFERENCES

1 Centers for Medicare \& Medicaid Services. Historical - National Health Expenditures Data. Available: https://www.cms.gov/ResearchStatistics-Data-and-Systems/Statistics-Trends-and-Reports/National HealthExpendData/NationalHealthAccountsHistorical.html [Accessed 20 July 2020].

2 Medpac.gov. 2020 report to the Congress: Medicare payment policy. Chapter 9: skilled nursing facility services. Available: http://www. medpac.gov/docs/default-source/reports/mar20_medpac_ch8_sec. pdf?sfvrsn $=0$ [Accessed 30 June 2020].

3 Bachmann S, Finger C, Huss A, et al. Inpatient rehabilitation specifically designed for geriatric patients: systematic review and meta-analysis of randomised controlled trials. BMJ 2010;340:c1718.

4 Hakkarainen TW, Arbabi S, Willis MM, et al. Outcomes of patients discharged to skilled nursing facilities after acute care hospitalizations. Ann Surg 2016;263:280-5.

5 Haley SM, Coster WJ, Andres PL, et al. Activity outcome measurement for postacute care. Med Care 2004;42:I-49.

6 Haley S, Jette A. RRTC for measuring rehabilitation outcomes: extending the frontier of rehabilitation outcome measurement and research. J Rehabil Outcome Meas 2000;4:31-41.

7 Blackwood J, Fernandez N. AM-PAC 6-Clicks scores predict hospital discharge destination in older adults with cardiovascular disease. Innov Aging 2018;2:489.

8 Covert S, Johnson JK, Stilphen M, et al. Use of the Activity Measure for Post-Acute Care "6 Clicks" Basic Mobility Inpatient Short Form and National Institutes of Health Stroke Scale to predict hospital discharge disposition after stroke. Phys Ther 2020;100:pzaa102.

9 Jette DU, Stilphen M, Ranganathan VK, et al. AM-PAC "6-Clicks" functional assessment scores predict acute care hospital discharge destination. Phys Ther 2014;94:1252-61.

10 Jette DU, Stilphen M, Ranganathan VK, et al. Validity of the AM-PAC "6-Clicks" inpatient daily activity and basic mobility short forms. Phys Ther 2014;94:379-91.

11 Jette DU, Stilphen M, Ranganathan VK, et al. Interrater reliability of AM-PAC "6-Clicks" basic mobility and daily activity short forms. Phys Ther 2015;95:758-66.

12 Menendez ME, Schumacher CS, Ring D, et al. Does "6-Clicks" day 1 postoperative mobility score predict discharge disposition after total hip and knee arthroplasties? J Arthroplasty 2016;31:1916-20.

13 Pfoh ER, Hamilton A, Hu B, et al. The Six-Clicks mobility measure: a useful tool for predicting discharge disposition. Arch Phys Med Rehabil 2020;101:1199-203.

14 Tymkew H, Norris T, Arroyo C, et al. The use of physical therapy ICU assessments to predict discharge home. Crit Care Med 2020;48:1312-8.

15 Virva R, Cistaro R, Moore A, et al. Clinical utility of the AM-PAC "6 Clicks" tools in hospital settings. Arch Phys Med Rehabil 2017;98:e43.

16 Borges PRT, Sampaio RF, Kirkwood RN, et al. Reduced version of the Activity Measure for Post-Acute Care (AM-PAC) for inpatients, "6-Clicks": Brazilian-Portuguese cross-cultural adaptation and measurement properties. Braz J Phys Ther 2020;24:231-9.

17 Geelen SJG, Valkenet K, Veenhof C. Construct validity and interrater reliability of the Dutch Activity Measure for Post-Acute Care "6-Clicks" basic mobility form to assess the mobility of hospitalized patients. Disabil Rehabil 2019;41:2563-9.

18 Hou W-H, Chiou H-Y, Chang F-H. Validation of the Mandarin version of the Activity Measure for Post-Acute Care (AM-PAC) "6-Clicks" among patients in acute rehabilitation. Am J Occup Ther 2020;74:7403205070p1.

19 Bose S, Brunty B, Spurrier T, et al. A Review of the Activity Measure for Post-Acute Care- "6 Clicks" (AM-PAC- "6-Clicks"). Crit Rev Phys Rehabil Med 2018;30:297-301.

20 Hiser S, Toonstra A, Friedman LA, et al. Inter-rater reliability of Activity Measure for Post-Acute Care '6-Clicks' inpatient mobility short form in the intensive care unit. Physiother Res Int 2020;25:e1849.

21 Hoyer EH, Young DL, Klein LM, et al. Toward a common language for measuring patient mobility in the hospital: reliability and construct validity of interprofessional mobility measures. Phys Ther 2018;98:133-42.

22 IBM Corp. IBM SPSS statistics, version 23.0. Armonk, NY: IBM Corp, 2015.

23 Burke RE, Jones CD, Coleman EA, et al. Use of post-acute care after hospital discharge in urban and rural hospitals. Am J Accountable Care 2017;5:16-22.

24 Dewhirst RC, Ellis DP, Mandara EA, et al. Therapists' perceptions of application and implementation of AM-PAC "6-Clicks" functional measures in acute care: qualitative study. Phys Ther 2016;96:1085-92. 